\title{
Psychological Approaches to Suicide Treatment and Prevention
}

\author{
David A. Jobes, Ph.D. ${ }^{*}$ \\ Josephine S. Au, B.A. \\ Asher Siegelman, B.A.
}

\author{
Address \\ "Department of Psychology, The Catholic University of America, 314 0'Boyle Hall, \\ Washington, DC, 20064, USA \\ Email: jobes@cua.edu
}

Published online: 5 October 2015

(C) Springer International Publishing AG 2015

This article is part of the Topical Collection on Suicide

Keywords Suicide treatment - Dialectical behavior therapy - Cognitive therapy for suicide prevention - The collaborative assessment and management of suicidality $\cdot$ Brief interventions

\section{Opinion statement}

In recent decades, the sub-specialization of "clinical suicidology" emphasizing suicide risk assessment, treatment, training, and the management of suicide-related liability has grown exponentially. This line of thinking had led to the development of suicide-specific treatments that target suicide as the focus of care (vs. a primary focus on treating mental disorders). These treatments are being extensively investigated using randomized controlled clinical trials to prove their efficacy and effectiveness. This article features the three main replicated treatments for suicide: Dialectical Behavior Therapy, Cognitive Therapy for Suicide Prevention, and the Collaborative Assessment and Management of Suicidality. In addition, there is a recent surge of brief suicide-focused interventions (1-4 sessions) that include variations of stabilization planning and close examination of suicide attempts as an opportunity to learn about suicidal risk with coping-oriented guidance and support. Within a rapidly evolving contemporary mental health care reality, these suicide-related treatments and interventions hold great promise for the prospect of providing more effective (and potentially life-saving care) for suicidal patients.

\section{Introduction}

In the wake of health care reform and dramatic changes in mental health and psychiatric care over recent decades, there has been an increasing focus on the topic of suicide risk within clinical practice and the professional literature $[1 \bullet, 2]$. This article will examine recent developments in psychological approaches to treating 
suicidality within the emerging specialty area of clinical suicidology. The specialized field of clinical suicidology covers areas including suicide risk assessment, clinical treatments for suicide risk, professional training in suicide-related practices, and issues of ethics and risk management related to suicidal risk. In this article, we aim to highlight two major domains within recent clinical treatment research. The first area will describe the replicated psychological treatments shown to be effective for suicidal risk through rigorous randomized controlled trials (RCTs). The second domain will consider exciting new clinical research focusing on the development of very brief (1-4 sessions) suicide-specific interventions. We will conclude with an integrated perspective on the field and next steps in clinical suicidology within contemporary mental health care.

\section{Evidence-based treatments for suicidal risk}

As discussed by Brown and Jager-Hyman [3], there are a number of RCTs for the treatment of suicide that have been conducted over the last three decades. There are many single studies showing non-significant findings along with some studies that show significant findings without needed replication. We will review three treatments with the most established empirical support. These psychological treatments for suicidal risk include the following: Dialectical Behavior Therapy (DBT), Cognitive Therapy for Suicide Prevention (CT-SP), and the Collaborative Assessment and Management of Suicidality (CAMS).

DBT is a form of cognitive-behavioral therapy (CBT) originally designed to treat suicidal and self-harming people who meet criteria for borderline personality disorder [4,5]. Bohus and colleagues [6] explain that DBT maintains the behavioral treatment components of skills training and change motivation, but there are components that make it distinct from traditional CBT. Specifically, DBT is a change-focused behavioral treatment; the dialectical approach of DBT utilizes an acceptance focus when a patient feels misunderstood and adopts a change focus when a patient needs motivation. The treatment is comprised of three distinct components: (a) skills training through structured individual or group therapy; (b) strengthening of skills and addressing barriers to motivation in individual psychotherapy; and (c) application of skills in everyday life through telephone contact with the psychotherapist. Furthermore, there is extensive team consultation for helping a therapist maintain focus as well as treatment adherence.

Since 1991, DBT has been tested in several RCTs, thus establishing its effectiveness over and above treatment as usual (TAU) for patients who meet criteria for borderline personality disorder (BPD), have engaged in selfinjurious behaviors, and/or have made suicide attempts [7•]. However, in an RCT in 2009, DBT was compared with another active, principle-based treatment approach (General Psychiatric Management) designed for people diagnosed with BPD [8]. A total of 180 patients diagnosed with BPD who had engaged in suicidal and non-suicidal self-injurious behaviors (at least twice in the last 5 years) were randomly assigned to one of the treatments. Both treatments produced significant reductions in suicidality and psychiatric symptoms, but there were no significant between-group differences, suggesting that DBT is not the only effective treatment approach for people with BPD who are at risk for 
suicide. Additionally, while the validity of DBT for suicidal behavior has been well established, its effectiveness to date has been largely restricted to female samples. This is significant due to the fact that males make up the largest percentage of completed suicides, with approximately $80 \%$ being males [9].

\section{Cognitive therapy for suicide prevention}

Cognitive Therapy for Suicide Prevention (CT-SP) is a cognitive-behavioral, suicide-specific, form of psychotherapy with strong empirical validation [10, 11]. The CT-SP approach endeavors to reduce risk factors for suicide and enhance coping with the elimination of suicidal behavior as the primary focus of the treatment [12]. Coping skills training in CT-SP targets patients' patterns of thinking, behavior, and interpersonal interactions that lead to suicidal states, referred to as the suicidal mode. A central tenet is that a suicidal person will continue to have stressors and problems that have triggered suicidal behavior in the past, but with effective coping skills, these stressors and problems will no longer function as triggers for suicidal behavior. The primary focus within this approach is to identify proximal triggers that put the patient into his/her suicidal mode. With this emphasis in mind, the goal is to address the most serious deficits the patient has in his/her coping abilities. Hence, patterns of thoughts, behaviors, and interpersonal interactions are addressed to strengthen these deficient areas. Techniques employed at the end of care include a novel relapse prevention strategy that uses guided imagery and the inventive use of a hope kit that serves as a memory aid for averting suicidal behavior.

From an empirical perspective, CT-SP was first investigated using a sample of 120 patients (61\% women) who had attempted suicide and were subsequently recruited from an emergency room following their attempts [10]. The investigators conducted an efficacy-based RCT comparing CT-SP and TAU over 10 individual treatment sessions. At an 18-month follow-up, they found that patients in CT-SP condition were $50 \%$ less likely than patients in TAU to make a repeat suicide attempt. The CT-SP patients were also found to be less depressed and more hopeful as a result of the treatment. In an important replication of the CT-SP intervention, Rudd and colleagues [13•] showed a $60 \%$ between-group decrease in suicide attempts within an RCT with 152 active-duty suicidal soldiers ( $>87 \%$ men) engaged in their brief cognitive therapy (BCBT) for the treatment of suicidal risk.

\section{Collaborative assessment and management of suicidality}

Another suicide-specific intervention with extensive empirical support is the Collaborative Assessment and Management of Suicidality (CAMS) $[14,15]$. CAMS is a suicide-specific therapeutic framework that is described as "non-denominational" in that a range of theoretical orientations and clinical techniques can be incorporated into the approach [16]. CAMS adopts a phenomenological approach to understanding a patient's suicidality, which leads to suicide-specific treatment planning that emphasizes the use of an outpatient stabilization plan and the identification and treatment of patient-defined suicidal "drivers" [17]. Within CAMS-guided care, collaboration is the key to enhancing the therapeutic alliance and motivating the patient as a pivotal member of the treatment team. Central to CAMS is the use of a multipurpose 


\section{Brief interventions for suicidal risk}

assessment, treatment planning, tracking, and outcome tool called the "Suicide Status Form" (SSF) that serves as a clinical road map and guides this suicide-specific intervention.

There are now seven non-randomized clinical trials $[14,16]$ and one RCT with 32 suicidal outpatients (62\% women) supporting the use of CAMS [18]. This RCT compared CAMS to enhanced care as usual (E$\mathrm{CAU}$ ) and found that CAMS patients improved significantly more in terms of suicidal ideation, overall symptom distress, hope, and patient satisfaction in comparison to E-CAU patients. There are now five current RCTs of CAMS underway around the world that will provide further data about the potential effectiveness of CAMS particularly in relation to self-harm and suicide attempt behaviors.

In response to rapid changes in contemporary mental health care delivery, there has been a recent surge of interest in brief suicide-specific clinical interventions [1•]. To fill emerging clinical needs, a cadre of innovative clinical suicidologists has been developing these brief interventions for suicide attempters that last for 1-4 sessions of intensive engagement and care.

There are various versions of stabilization-oriented clinical interventions that aimed to be the remedy for "no-harm" or "no-suicide" contracting. Some of the more prominent of these are Safety Planning, Crisis Response Planning, and the SSF Stabilization Plan that is used within CAMS. Probably the best known of these interventions is the Safety Planning Intervention (SPI) developed by Stanley and Brown [19]. This intervention was created to increase treatment engagement and to develop suicide-specific coping strategies among a range of suicidal patients seen in a variety of clinical settings. A typical Safety Plan Intervention lasts about 20$45 \mathrm{~min}$, during which the clinician and patient identify warning signs that might precipitate a suicidal crisis. The dyad then proceeds to develop a six-step hierarchical list of internal coping strategies, and external sources of social and professional support should the patients' suicidal thoughts emerge. Additionally, clinicians are also recommended to evaluate the likelihood of which patients will use the safety plan as well as to incentivize them to do so. The SPI has been adapted in several settings. For instance, it has been tested on veterans presented to EDs and in a psychiatric inpatient setting for suicide-related issues [20, 21]. One recent study has shown that SPI, when combined with a structured follow-up, is related to increased treatment attendance and decreased hospitalization 3 months postemergency department discharge [22].

In a related line of thinking and clinical research, Bryan and colleagues [23] are studying the use of "Crisis Response Planning" combined with the development of "Reasons for Living" as a brief one-session intervention for suicidal patients. This intervention is being investigated in a RCT with active-duty military service members and reflects a related but elaborated form of suicidespecific stabilization planning within this emerging genre of brief interventions. 
O'Connor and colleagues [24] developed the "Teachable Moment Brief Intervention" (TMBI) which aims to foster change during a key window of opportunity following a suicide attempt [25]. The TMBI follows research showing that cueing events are subjected to different interpretations and thus can create the opportunity to increase motivation to change risky health behaviors [24]. The TMBI is partly inspired by the collaborative philosophy of CAMS and the functional analysis approach used for understanding self-harm behaviors in DBT [25]. It focuses on building rapport, identifying factors underlying a suicide attempt, crisis planning, and planning for outpatient mental health services. Preliminary evidence shows that TMBI was well received by patients at a level 1 trauma center and that those in the TMBI group showed a consistent linear trend in their awareness as well as readiness to change when compared to the TAU group. The TMBI is currently being investigated in larger clinical trial research.

\section{Motivational interviewing for suicidal ideation}

Motivational interviewing is a patient-centered approach used to foster a patients' intrinsic desire to change their health behaviors by resolving ambivalence and it has recently been adapted for addressing suicide ideation [26-28]. Based on Kovocs and Beck's internal struggle hypothesis [29] (which postulates that suicide is a result of the inner struggle between one's wish to live and wish to die), the goal of the motivational interviewing to address suicide ideation (MI-SI) is to increase patients' motivation to live, so as to reduce their overall risk of suicide [27]. In other words, it carefully directs a patient's motivation to die in the direction of living with the goal of making life more worth living [28]. Researchers of MI believe that discussion of suicide unnecessarily leads patients to thinking about suicide; therefore, they advise clinicians to focus on change talk about living instead of suicide talk [30]. It is also recommended that MI-SI should be used as an adjunct to other treatment instead of as a standalone treatment. The results of an open trial of MI-SI, which is comprised of two sessions that span in 3 days, has shown that patients experienced a reduction of suicide ideation immediately as well as 2 months after treatment when compared to baseline measures [26].

\section{Attempted suicide short intervention program}

Another brief intervention that has been recently tested within a RCT is called the Attempted Suicide Short Intervention Program (ASSIP) [31]. The ASSIP is comprised of three sessions, each of which lasts about 60 to $90 \mathrm{~min}$. In the first session, patients are asked to narrate their stories to the clinician that led up to their suicide attempts, which are video recorded and then played back to the dyad in the following session. The goal of this is to reenact the progression of their suicidal crisis in a controlled environment, so that the clinical dyad can create a psychoeducational handout by the end of the second session. In the third session, the clinician presents a case conceptualization that is revised collaboratively with the patient; a list of warnings signs and individualized safety strategies are given to the patient to keep with them. 


\section{Conclusions}

In a 2-year follow-up of the RCT ( $n=120)$, the ASSIP group was found to have an $80 \%$ reduction in repeated suicide attempts when compared to the TAU group [31]. Additionally, those in the ASSIP group spent significantly fewer days within psychiatric hospital care.

These are exciting times for the field of clinical suicidology; the field is rapidly evolving with a record number of suicide-focused RCTs underway around the world [32]. Major changes within contemporary health care (e.g., health care reform in the United States) are fostering significant innovations in mental health care delivery, with a particular emphasis on managing suicide risk [1•]. It follows that there is an increasing emphasis on least-restrictive, evidence-based, and cost-effective suicide-specific clinical care. The sheer economics of clinically dealing with suicide and suicide-related morbidity is a major force that is leading to changes in caring for suicidal patients. As discussed elsewhere [33], an evolving "stepped-care" model for treating suicidal risk may well emerge in the coming years that creates a continuum of clinical care ranging from the paraprofessional and peer-based support, to suicide-specific care involving brief interventions, as well as suicidespecific outpatient, partial, and respite clinical care. Likewise, there is an emerging interest in stratifying the risk of different suicidal states with the potential for matching different kinds and doses of treatments to different suicidal states [34,35]. Given the known high risk of suicide that follows inpatient discharge [36], inpatient psychiatric care will increasingly need to embrace the use of suicide-specific interventions during an inpatient stay with an emphasis on safety planning and postdischarge means-restriction along with thoughtful disposition planning that bridges a patient to effective outpatient care. There may be value in further tailoring suicidal treatments in gender-specific ways. In the coming years, we are likely to see more use of suicide-specific group modalities [37] and the increasing use of technology applications for clinically monitoring, managing, and even treating suicidal risk [33, 38].

When we reflect on the evidence-base for effectively treating suicidality, we are struck by some remarkably common themes that unite the treatments and interventions described in this review. For example, all of the interventions we have discussed employ various means for helping a suicidal patient recognize the when, how, where, and why they become suicidal. Once the patient is therapeutically "trained" to recognize the nature and occurrence of their suicidal risk, they are similarly taught to use suicide-specific coping skills and techniques to help avert suicidal behaviors. All these interventions tend to be non-adversarial and collaborative in nature, emphasizing the importance of empathy, understanding, and empathic validation of a patient's suicidal state. All the described interventions in this article endeavor to inspire, harness, and channel the patient's motivation, so as to better cope with their suicidal impulses and to decrease the suicidal risk therein. Finally, for the most part, all these interventions either directly or implicitly attend to existential aspects inherent to the suicidal struggle with a marked emphasis on the psychological importance 
of hope and the pursuit of a life worth living. Given the increasing number of suicide-focused treatment investigations that are now underway, we look forward to the promise of suicidal patients receiving more effective evidence-based clinical care in the years to come.

\section{Compliance with Ethical Standards}

\section{Conflict of Interest}

David A. Jobes reports grants from NIMH, DOD, and AFSP; he receives book royalties from the American Psychological Assoc Press, The Guilford Press, and e-learning royalties from Empathos Resources. Dr. Jobes is Co-Owner of CAMS-care, LLC. Josephine S. Au and Asher Siegelman declare that they have no conflict of interest.

\section{Human and Animal Rights Informed Consent}

No human or animal studies performed by the authors:

This article does not contain any studies with human or animal subjects performed by any of the authors.

\section{References and Recommended Reading}

Papers of particular interest, published recently, have been highlighted as:

- Of importance

1. Jobes DA, Bowers ME. Treating suicidal risk in a posthealthcare reform era. J Aggress Confl Peace Res. 2015;7:167-78.

This article provides a brief introduction to evidence-based treatments for suicidal patients and illustrates multiple advantages/benefits in utilizing these treatments. A significant overview of CAMS and CAMS research is provided.

2. Jobes DA, Rudd MD, Overholser JC, Joiner Jr TE. Ethical and competent care of suicidal patients: contemporary challenges, new developments, and considerations for clinical practice. Prof Psychol. 2008;39:405.

3. Brown GK, Jager-Hyman S. Evidence-based psychotherapies for suicide prevention: future directions. Am J Prev Med. 2014;47:S186-94.

4. Linehan M. Cognitive-behavioral treatment of borderline personality disorder. New York: Guilford Press; 1993.

5. Linehan MM, Armstrong HE, Suarez A, Allmon D, Heard HL. Cognitive-behavioral treatment of chronically parasuicidal borderline patients. Arch Gen Psychiatry. 1991;48:1060-4.

6. Bohus M, Haaf B, Stiglmayr C, Pohl U, BoÈhme R, Linehan M. Evaluation of inpatient dialecticalbehavioral therapy for borderline personality disorder-a prospective study. Behav Res Ther. 2000;38:875-87.
7. Linehan MM, Korslund KE, Harned MS, Gallop RJ, Lungu A, Neacsiu AD, et al. Dialectical behavior therapy for high suicide risk in individuals with borderline personality disorder: a randomized clinical trial and component analysis. JAMA Psychiatry. 2015;72:475-82.

This article highlights the consistent efficacy of DBT for treating suicidal women with BPD. It also provides an explanation of the treatment utility of distinct components in the DBT protocol.

8. McMain SF, Links PS, Gnam WH, Guimond T, Cardish RJ, Korman L, et al. A randomized trial of dialectical behavior therapy versus general psychiatric management for borderline personality disorder. JAMA Psychiatry. 2009; 166:1365-74.

9. Currier GW, Brown GK, Brenner LA, Chesin M, Knox KL, Ghahramanlou- Drapeau CW, et al. U.S.A. Suicide 2012: Official Final Data. American Association of Suicidology. October 18, 2014. Available at http:// www.suicidology.org. Accessed 20 Jul 2015

10. Brown GK, Ten Have T, Henriques GR, Xie SX, Hollander JE, Beck AT. Cognitive therapy for the prevention of suicide attempts: a randomized controlled trial. JAMA. 2005;294:563-70.

11. Wenzel A, Brown GK, Beck AT, editors. Cognitive therapy for suicidal patients: scientific and clinical applications. Washington, DC: American Psychological Association; 2009. 
12. Stanley B, Brown G, Brent DA, Wells K, Poling K, Curry $\mathrm{J}$, et al. Cognitive-behavioral therapy for suicide prevention (CBT-SP): treatment model, feasibility, and acceptability. J Am Acad Child Adolesc Psychiatry. 2009;48:1005-13.

13. Rudd MD, Bryan CJ, Wertenberger EG, Peterson AL, Young-McCaughan S, Mintz J, et al. Brief cognitive-behavioral therapy effects on post-treatment suicide attempts in a military sample: results of a randomized clinical trial with 2-year follow-up. Am J Psychiatr. 2015;172:441-9.

This article provides current empirical research efficacy of CBT for suicide prevention including a description of the CBT protocol.

14. Jobes DA. The Collaborative Assessment and Management of Suicidality (CAMS): an evolving evidence-based clinical approach to suicidal risk. Suicide Life Threat Behav. 2012;42:640-53.

15. Jobes DA. Managing suicidal risk: a collaborative approach. New York: Guilford Press; 2006.

16. Jobes DA, Comtois KA, Brenner LA, Gutierrez PM, $\mathrm{O}^{\prime}$ Connor SS. Lessons learned from clinical trials of the Collaborative Assessment and Management of Suicidality (CAMS). In: O'Connor RC, Pirkis, J. editors. International handbook of suicide prevention: research, policy, \& practice. 2nd ed. West Sussex: WileyBlackwell; 2015. in press.

17. Tucker RP, Crowley KJ, Davidson CL, Gutierrez PM. Risk factors, warning signs, and drivers of suicide: what are they, how do they differ, and why does it matter? Suicide Life Threat Behav. 2015. doi:10.1111/sltb.12161.

18. Comtois KA, Jobes DA, O'Connor S, Atkins DC, Janis $\mathrm{K}$, Chessen $\mathrm{E}$, et al. Collaborative assessment and management of suicidality (CAMS): feasibility trial for next-day appointment services. Depress Anxiety. 2011;28:963-72.

19. Stanley B, Brown GK. Safety Planning Intervention: a brief intervention to mitigate suicide risk. Cogn Behav Pract. 2012;19:256-64.

20. Currier GW, Brown GK, Brenner LA, Chesin M, Knox KL, Holloway MG, et al. Rationale and study protocol for a two-part intervention: safety planning and structured follow-up among veterans at risk for suicide and discharged from the emergency department. Contemp Clin Trials. 2015;43:179-84.

21. Rings JA, Alexander PA, Silvers VN, Gutierrez PM. Adapting the Safety Planning Intervention for use in a veterans psychiatric inpatient group setting. J Ment Health Couns. 2012;34:95-109.

22. Stanley B, Brown GK, Currier GW, Lyons C, Chesin M, Knox KL. Brief intervention and follow-up for suicidal patients with repeat emergency department visits enhances treatment engagement. Am J Public Health. 2015. doi:10.2105/AJPH.2015.302656.

23. Bryan CJ, Rudd MD. Brief interventions to prevent suicide attempts in military personnel. In: Bryan CJ, Rudd MD, Bajor L, Hoge C, Sickel M, editors. Issues in the care of military personnel and veterans. Paper presented at the annual meeting of the American

Psychiatric Association, Toronto, Canada; 2015.
24. O'Connor SS, Comtois KA, Wang J, Russo J, Peterson R, Lapping-Carr L, et al. The development and implementation of a brief intervention for medically admitted suicide attempt survivors. Gen Hosp Psychiatry. 2015. doi:10.1016/j.genhosppsych.2015.05.001.

25. McBride CM, Emmons KM, Lipkus IM. Understanding the potential of teachable moments: the case of smoking cessation. Health Educ Res. 2003;18:156-70. 26. Britton PC, Conner KR, Maisto SA. An open trial of motivational interviewing to address suicidal ideation with hospitalized veterans. J Clin Psychol.

2012;68:961-71.

27. Britton PC, Patrick H, Wenzel A, Williams GC. Integrating motivational interviewing and self-determination theory with cognitive behavioral therapy to prevent suicide. Cogn Behav Pract. 2011;18:1627.

28. Britton PC, Williams GC, Conner KR. Selfdetermination theory, motivational interviewing, and the treatment of clients with acute suicidal ideation. J Clin Psychol. 2008;64:52-66.

29. Kovacs M, Beck AT. The wish to die and the wish to live in attempted suicides. J Clin Psychol. 1977;33:361-5.

30. Britton PC. Motivational interviewing to address suicidal ideation. In: Arkowitz H, Miller WR, Rollnick, editors. Motivational interviewing in the treatment of psychological problems. New York: Guilford Press; 2015.

31. Gysin-Maillart A, Soravia L, Megert M, Schwab S, Michel K. A novel brief therapy for attempted suicide: two year follow-up randomized controlled study of the Attempted Suicide Short Intervention Program (ASSIP). Manuscript submitted for publication; 2015. 32. Jobes DA. Reflections on suicide among soldiers. Psychiatry. 2013;76:126-31.

33. Jobes DA. Innovations in suicide risk assessment and crisis intervention. Invited plenary address to the Congress of the International Association for Suicide Prevention; Oslo, Norway; September, 2013.

34. Brown GK, Steer RA, Henriques GR, Beck AT. The internal struggle between the wish to die and wish to live: a risk factor for suicide. Am J Psychiatry. 2005; 162:1977-9.

35. Corona CD, Jobes DA, Nielsen AC, Pedersen CM, Jennings KW, Lento RM, et al. Assessing and treating different suicidal states in a Danish outpatient sample. Arch Suicide Res. 2013;17:302-12.

36. Meehan J, Kapur N, Hunt IM, Turnbull P, Robinson J, Bickley $\mathrm{H}$, et al. Suicide in mental health in-patients and within 3 months of discharge. Br J Psychiatry. 2006;188:129-34.

37. Johnson LL, O'Connor SS, Kaminer B, Jobes DA, Gutierrez PM. Suicide-focused group therapy for veterans. Mil Behav Health. 2014;2:327-36.

38. Bush NE, Dobscha SK, Crumpton R, Denneson LM, Hoffman JE, Crain A, et al. A virtual hope box smartphone app as an accessory to therapy: proof-ofconcept in a clinical sample of veterans. Suicide Life Threat Behav. 2015;24:1-9. 